\title{
Sonographic findings associated with stenosis progression and vascular complications in moyamoya disease
}

\author{
Woo-Jin Lee, MD, ${ }^{1}$ Keun-Hwa Jung, MD, PhD, ${ }^{1,2}$ Keon-Joo Lee, MD, ${ }^{1}$ Jeong-Min Kim, MD, PhD, ${ }^{3}$ \\ Soon-Tae Lee, MD, PhD, ${ }^{1,2}$ Kon Chu, MD, PhD, ${ }^{1,2}$ Sang Kun Lee, MD, PhD, ${ }^{1,2}$ and \\ Jae-Kyu Roh, MD, PhD ${ }^{1,4}$
}

'Department of Neurology, Seoul National University Hospital; 2Program in Neuroscience, Neuroscience Research Institute of SNUMRC, College of Medicine, Seoul National University; ${ }^{3}$ Department of Neurology, Chung-Ang University Hospital, Seoul; and ${ }^{4}$ Department of Neurology, The Armed Forces Capital Hospital, Sungnam, South Korea

\begin{abstract}
OBJECTIVE The progression of arterial stenosis in patients with moyamoya disease (MMD) has variable courses and an unclear mechanism. The authors hypothesized that elevated wall shear stress (WSS) at the terminal internal carotid artery (ICA) and proximal middle cerebral artery (MCA) may facilitate MMD progression. They indirectly evaluated the relative magnitude of WSS (WSS value [WSSV]) with MR angiography (MRA) and transcranial Doppler to determine its predictive value for stenosis progression (SP) and the development of vascular complications.
\end{abstract}

METHODS Thirty-one medically treated patients (58 hemispheres and 95 nonoccluded vessels) were analyzed with serial MRA (median follow-up 23 months). The parameters studied were SP, SP rates (SPRs) for individual ICAs/MCAs, and their mean values from the ipsilateral hemispheres as mean SP (MSP) and MSP rates (MSPRs). Significant progression was defined as decrements of $\geq 20 \%$ for SP and MSP and $\geq 10 \% /$ year for SPR and MSPR. The development of vascular complications in relevant hemispheres was also recorded. The WSSV (dyne/ $\left.\mathrm{cm}^{2}\right)$ was defined as the shear rate multiplied by blood viscosity.

RESULTS After adjusting the initial stenosis degree and MRA stage of MMD, an SP of $\geq 20 \%$ and an SPR of $\geq 10 \%$ / year were associated with the highest-quartile WSSVs for all individual vessels and for MCAs and ICAs separately. For each hemisphere, an MSP of $\geq 20 \%$ and an MSPR of $\geq 10 \%$ /year were associated with the highest-quartile mean WSSVs. Furthermore, significant SP was highly correlated with vascular complications, and the highest-quartile mean WSSV was independently associated with vascular complications in relevant hemispheres.

CONCLUSIONS An elevated WSSV is an independent predictor for SP and vascular complications in nonoccluded MMD.

http://thejns.org/doi/abs/10.3171/2015.6.JNS15933

KEY WORDS moyamoya disease; transcranial Doppler; angiography; progression; vascular remodeling; wall shear stress; vascular disorders

$\mathrm{M}$ OYAMOYA disease (MMD) is an idiopathic, progressive arteriopathy of the terminal internal carotid artery (ICA) and proximal portions of its branches, including the middle cerebral artery (MCA) and the anterior cerebral artery. ${ }^{8,16,25}$ Stenosis progression (SP) in patients with MMD occurs frequently. ${ }^{17}$ Various factors regarding vascular remodeling, including matrix metalloproteinases (MMPs) and their natural inhibitors (tissue inhibitors of metalloproteinases [TIMPs]), are known to be altered in MMD, 2,5,8,13,14,16,19,25,30 implying that dysregulation of the vascular remodeling response to various stressors could be the mechanism of SP. ${ }^{8,16,25}$ Furthermore,

ABBREVIATIONS BV = blood viscosity; $\mathrm{DR}=$ diameter ratio; $I C A=$ internal carotid artery; $I Q R=$ interquartile range; $M C A=$ middle cerebral artery; $M F V=$ mean flow velocity; $\mathrm{MMD}=$ moyamoya disease; $\mathrm{MMP}=$ matrix metalloproteinase; $\mathrm{MRA}=\mathrm{MR}$ angiography; $\mathrm{MSP}=$ mean stenosis progression; $\mathrm{MSPR}=\mathrm{MSP}$ rate; $\mathrm{MWSSV}=$ mean wall shear stress value; $\mathrm{ROC}=$ receiver operating characteristic; $\mathrm{SD}=$ stenosis degree; $\mathrm{SP}=$ stenosis progression; $\mathrm{SPR}=\mathrm{SP}$ rate; $\mathrm{SR}=$ shear rate; $\mathrm{TCD}=$ transcranial Doppler; TIMP = tissue inhibitor of metalloproteinase; TOF = time-of-flight; WSS = wall shear stress; WSSV = WSS value.

SUBMITTED April 24, 2015. ACCEPTED June 4, 2015.

INCLUDE WHEN CITING Published online December 11, 2015; DOI: 10.3171/2015.6.JNS15933. 
elevated endothelial wall shear stress (WSS), a force generated by the blood flow nearest to the vessel wall and imposed on the vascular endothelium, can significantly alter the levels and activities of these factors and consequently promote vascular remodeling. 4,6,23,26 Considering that the WSS is highest at the primary pathological sites of MMD, the terminal ICA and its proximal branches, we hypothesized that elevated WSS may play an important pathophysiological role in the progression of MMD..$^{14,29}$

In terms of measuring the magnitude of WSS, numerous studies indirectly calculated the relative WSS value (WSSV) with sonography by measuring arterial diameter and centerline flow velocity. ${ }^{10,11}$ Transcranial Doppler (TCD) measures the velocity of blood flow in basal cerebral arteries and can estimate arterial stenosis., ${ }^{1,28,31}$ Therefore, TCD can be useful in diagnosing and staging MMD.$^{18}$ TCD also precisely locates the site of arterial stenosis, so we can estimate the shear rate (SR) at a relevant site by using both TCD and MR angiography (MRA). In this study, we hypothesized that for nonoccluded vessels in patients with MMD, an elevated WSSV could be an independent predictor for SP. Therefore, we evaluated WSSVs with TCD and MRA to determine their relationship with SP and vascular complications.

\section{Methods \\ Study Population and Follow-Up}

We identified 106 patients older than 18 years who were diagnosed with MMD between 2003 and 2012 at Seoul National University Hospital. MMD was diagnosed by digital subtraction angiography according to current diagnostic criteria. ${ }^{15}$ To evaluate the factors associated with arterial stenosis, patients who met the following conditions were subjected to further analysis: 1) at least 1 vessel of the distal ICA and the proximal MCA of each hemisphere not occluded; 2) baseline MRI/MRA and TCD evaluations with an interval of less than 2 weeks; 3 ) favorable temporal windows for TCD and adequate TCD data; 4) follow-up MRI/MRA with an interval of more than 12 months; 5) no other potential causes of moyamoya syndrome; and 6) no surgical treatment for MMD until the follow-up MRI/MRA (because such a surgery might have significantly altered the hemodynamics of basal cerebral arteries). As a result, 19 patients with complete occlusion of both the MCA and the ICA, 26 patients with inadequate follow-up MRI/MRA, and 30 patients who had received surgical treatment were excluded. Thirty-one patients (58 hemispheres and 95 nonoccluded moyamoya vessels [57 ICAs and $38 \mathrm{MCAs}]$ ) were enrolled in our study. Clinical, laboratory, demographic, radiographic, and sonographic profiles of each patient were compiled. Vascular complications, such as cerebral hemorrhage and ischemia, that were confirmed by MRI were noted also. Approval to conduct this study was received from the Seoul National University Hospital Institutional Review Board. All medical records/information were anonymized and deidentified before analysis.

\section{Analysis MRI and MRA}

Initial and follow-up MRI/MRA was performed by us- ing either a 1.5-T or a 3.0-T imaging system (Siemens Verio) according to an MRI protocol that included FLAIR, gradient echo, time-of-flight (TOF) angiography, and contrast-enhanced MRA with or without diffusion-weighted imaging. Stages of arterial stenosis in each hemisphere were scored from 0 to 10 by summing the scores of the ICA, MCA, anterior cerebral artery, and posterior cerebral artery according to a method introduced by Houkin et al. ${ }^{9}$ ( 0 , normal; 1 , stenosis of $\mathrm{C}_{1} / \mathrm{M}_{1} ; 2$, focal occlusion of $\mathrm{C}_{1} /$ $\mathrm{M}_{1}$; and 3, total nonvisualization of $\mathrm{C}_{1} / \mathrm{M}_{1}$ [for the ICA and MCA]; or 0, normal; 1, stenosis/focal occlusion of $\mathrm{A}_{2} /$ $\mathrm{P}_{2}$; and 2, total nonvisualization of $\mathrm{A}_{2} / \mathrm{P}_{2}$ [for the anterior cerebral artery and posterior cerebral artery]). To evaluate the degree of stenosis precisely, we measured the arterial diameter of each nonoccluded vessel in the 4-timesmagnified view of TOF angiography sequences at the most stenosed portion in the proximal $\mathrm{M}_{1}$ segment for the MCA and the ophthalmic segment $\left(\mathrm{C}_{6}\right)$ for the ICA. Source images of TOF MRI sequences were also reviewed. Because vessel stenosis in patients with MMD is longitudinal, the diameter ratio (DR) was adopted for evaluating the relative degrees of stenosis. The DR was calculated by dividing the arterial diameter by the reference value, which is the proximal basilar artery diameter evaluated at the level just distal to the anterior inferior cerebellar artery branching. ${ }^{24}$ The stenosis degree (SD) was calculated by subtracting the DR from $1(\mathrm{SD}=1-\mathrm{DR})$. The factors for disease progression were defined as follows: SP (percentage) as the increment of SD from the initial visit to the follow-up, and SP rate (SPR [percentage/year]) as SP divided by the time interval between the initial and follow-up images. The mean values of these factors from the ipsilateral hemispheres (ICAs/ MCAs) were also calculated to evaluate the integrated SP of each hemisphere as follows: mean SP (MSP [percentage]) and MSP rate (MSPR [percentage/year]). If one of the vessels was occluded, values from the remaining nonoccluded vessel were regarded as the mean values. Significant SP was defined for each parameter as follows: a progression of $20 \%$ or more for $\mathrm{SP}(\mathrm{SP} \geq 20 \%)$ and MSP (MSP $\geq 20 \%$ ) and a progression of $10 \%$ or more per year for the $\mathrm{SPR}$ (SPR $\geq 10 \% /$ year) and MSPR (MSPR $\geq 10 \% /$ year).

\section{Sonographic Analysis}

Basal cerebral arteries were evaluated sonographically by a 2-MHz pulsed Doppler instrument according to standard protocol. The studies were conducted by a skilled sonographer and read by 2 stroke specialists who were blinded to the clinical data. TCD data, including mean flow velocity (MFV $[\mathrm{cm} / \mathrm{sec}]$ ) and pulsatility index, were obtained at the level of the distal portion of the ICA (from a transorbital window with an insonation depth of 65-80 $\mathrm{mm}$, by identifying a receding flow proximal to the ICA bifurcation) and the proximal portion of the MCA (from a temporal window with an insonation depth of $65-70 \mathrm{~mm}$, by identifying an approaching flow approximately 10-15 $\mathrm{mm}$ distal to the ICA bifurcation). ${ }^{22}$

\section{Evaluation of Endothelial Shear Stress}

The WSSV $\left(\right.$ dyne $/ \mathrm{cm}^{2}$ ) was calculated by multiplying the SR and blood viscosity (BV). Assuming that the termi- 
nal ICA and proximal MCA are circular pipes and that the velocity profile of the blow flow is parabolic, SR $(\gamma)$ could be defined as follows:

$$
\gamma\left(\sec ^{-1}\right)=8 \times \mathrm{MFV} / \mathrm{D},
$$

where MFV and D are the mean flow velocity and lumen diameter, respectively. ${ }^{11}$ For BV $(\mu)$, considering the shear thinning effect of blood flow, we adopted the Carreau-Yasuda model:

$$
\mu(\text { in } \mathrm{cP})=\mu_{\infty}+\left(\mu_{0}-\mu_{\infty}\right)\left[1+\left(\lambda_{\text {cy }} \gamma\right)^{\mathrm{a}}\right]^{(\mathrm{n}-1) / \mathrm{a}},
$$

where $\mu$ is the viscosity for SR $\gamma ; \mu_{\infty}$ is the viscosity for infinite SR; $\mu_{0}$ is the viscosity at 0 SR; and $\lambda_{\text {cy }}$, a, and $n$ are fitting parameters. ${ }^{3}$ For a valid blood-mimicking fluid, KSCN-X solution, these parameters have the following values: $\mu_{0}=22 \mathrm{cP} ; \mu_{\infty}=2.2 \mathrm{cP} ; \mathrm{a}=0.644 ; \mathrm{n}=0.392 ;$ and $\lambda_{\text {cy }}$ $=0.110 \mathrm{sec}$. Given that the BV increases exponentially with increasing hematocrit levels, correction for the variations in $\mathrm{BV}$ according to the hematocrit level is necessary. We used the method proposed by Box et al. ${ }^{3}$ to replace the parameter $\mathrm{n}$ with the hematocrit level. Mean WSSVs from the ipsilateral ICAs/MCAs were also calculated and defined as mean WSSV (MWSSV) (dyne/ $\left.\mathrm{cm}^{2}\right)$. The Reynolds number was calculated according to the following equation:

$$
\mathrm{R}=(\rho \times \mathrm{MFV} \times \mathrm{r}) / \mu,
$$

where $\rho$ is the blood density (assumed to be $1.060 \times 10^{3}$ $\left.\mathrm{kg} / \mathrm{m}^{3}\right), \mathrm{MFV}$ is the mean velocity of the blood $(\mathrm{m} / \mathrm{sec}), \mathrm{r}$ is the radius of the vessel (mm), and $\mu$ is the BV (cP). A Reynolds number of $<1000$ is usually considered characteristic of laminar flow.? The luminal irregularity, or curvature of the vessels, was not taken into account in the evaluation of the WSSV.

\section{Statistical Analysis}

IBM SPSS Statistics version 21.0 for Windows was used for statistical analysis. Student t-tests were conducted for interval variables, and chi-square tests were used for dichotomous or qualitative variables. Variables with a $p$ value $<0.10$ were included in the multivariate analysis. For multivariate analysis, a logistic regression test was used. A receiver operating characteristic (ROC) curve was used to evaluate the efficacy of the WSSV to predict SP. Statistical significance was set at $\mathrm{p}<0.05$.

\section{Results}

\section{Characteristics of the Study Population}

The median follow-up duration was 23 months (range 12-60 months, interquartile range [IQR] 15-35 months). Eight patients $(25.8 \%)$ were male, and the mean age was $46.0 \pm 11.5$ years. Clinical and demographic profiles are listed in Table 1. For the 58 hemispheres, the initial mean SD ( \pm standard deviation) was $27.1 \% \pm 17.8 \%$. The median MRA stages were 3 (IQR 1-4) for each hemisphere and 5 (IQR 4-8) for the sum of bilateral hemispheres. The median follow-up MRA stage was 4 (IQR 2-5), and progression of stage was noted in 9 patients $(15.5 \%)$ for each hemisphere. Ten vascular complications (17.2\%; 7 ische-
TABLE 1. Demographic and laboratory data $(n=31)^{*}$

\begin{tabular}{lc}
\hline Demographic or Lab Info & Value \\
\hline Age in yrs & $46.0 \pm 11.5$ \\
\hline Male sex & $8(25.8)$ \\
\hline Hypertension & $10(32.3)$ \\
\hline Diabetes & $2(6.5)$ \\
\hline Hyperlipidemia & $4(12.9)$ \\
\hline Smoking & $1(3.2)$ \\
\hline HbA1c $(\%)$ & $5.7 \pm 0.5$ \\
\hline Total cholesterol $(\mathrm{mg} / \mathrm{dl})$ & $170.0 \pm 28.4$ \\
\hline LDL cholesterol $(\mathrm{mg} / \mathrm{dl})$ & $98.7 \pm 28.9$ \\
\hline Hematocrit $(\%)$ & $39.9 \pm 4.1$ \\
\hline
\end{tabular}

$\mathrm{HbA1c}=$ glycated hemoglobin; Info = information; LDL = low density lipoprotein.

* Data are reported as mean \pm standard deviation or number (\%).

mia and 3 hemorrhage) developed during follow-up with a mean delay of $21.9 \pm 9.7$ years (Table 2 ).

\section{Stenosis Progression}

For each vessel, the initial mean SD was $38.0 \pm 17.8$ $(20.2 \pm 18.7$ for ICAs, $42.7 \pm 21.9$ for MCAs). The SP and SPR were $5.9 \% \pm 21.8 \%(0.01 \% \pm 19.09 \%$ for ICAs, $14.65 \% \pm 23.08 \%$ for MCAs) and $3.5 \% \pm 17.2 \% /$ year $(0.21 \% \pm 15.29 \% /$ year for ICAs, $8.52 \% \pm 19.01 \% /$ year for MCAs), respectively. Significant SP for each parameter was reported as follows: 22 (23.2\%) for an SP $\geq 20 \%$ (7 [12.3\%] for ICAs, 15 [39.5\%] for MCAs) and 26 (27.4\%) for an SPR $\geq 10 \% / y e a r(10$ [17.5\%] for ICAs, 16 [42.1\%] for MCAs). For each hemisphere, the MSP and MSPR were $5.8 \% \pm 16.1 \%$ and $3.4 \% \pm 12.4 \% /$ year, respectively. Significant SP for each parameter was as follows: $10(17.2 \%)$ for an MSP $\geq 20 \%$ and 12 (20.7\%) for an MSPR $\geq 10 \% /$ year. MCAs showed more severe initial SD and more frequent significant SP than ICAs (Tables 2 and 3).

TABLE 2. Radiological profiles for hemispheres*

\begin{tabular}{lc}
\hline \multicolumn{1}{c}{ Variable } & Value \\
\hline MWSSV $\left(\right.$ dyne $\left./ \mathrm{cm}^{2}\right)$ & $13.5 \pm 7.3$ \\
\hline Initial mean SD $(\%)$ & $27.1 \pm 17.8$ \\
\hline MRA stage & $3(1-4)$ \\
\hline MRA stage $>3$ & $36(62.0)$ \\
\hline Follow-up mean SD $(\%)$ & $32.6 \pm 19.4$ \\
\hline Follow-up MRA stage & $4(2-5)$ \\
\hline MRA stage progression & $9(15.5)$ \\
\hline MSP $(\%)$ & $5.8 \pm 16.1$ \\
\hline MSP $\geq 20 \%$ & $10(17.2)$ \\
\hline MSPR (\%/yr) & $3.4 \pm 12.4$ \\
\hline MSPR $\geq 10 \% / y r$ & $12(20.7)$ \\
\hline Hemorrhage & $3(5.2)$ \\
\hline Ischemia & $7(12.1)$ \\
\hline
\end{tabular}

* Data are reported as mean \pm standard deviation, median (IQR), or number $(\%)$ of hemispheres $(n=58)$. 


\section{Profiles of WSS}

In the sonographic analyses, the MWSSVs were $12.3 \pm$ $8.5 \mathrm{dyne} / \mathrm{cm}^{2}$ for ICAs and $15.4 \pm 9.7 \mathrm{dyne} / \mathrm{cm}^{2}$ for MCAs, and the MWSSV for each hemisphere was $13.5 \pm 7.3$ dyne/ $\mathrm{cm}^{2}$ (Tables 2 and 3). The Reynolds number ranged from 35.0 to 683.0 , indicating that blood flows could be regarded as laminar. To evaluate the effect of an elevated WSSV in the progression of vessel stenosis, we selected vessels/ hemispheres of which the WSSV/MWSSV were in the highest-quartile group (highest-quartile WSSV/MWSSV). To adjust the effect of initial SD with the WSSV or the progression of vessel stenosis, we evaluated the relations of progression factors with factors such as having an initial SD value higher than the median (upper-half SD) or higher than the third quartile (highest-quartile SD) and advanced hemispheric MRA stage (MRA stage $\geq 4$ ), and we adjusted them for later analyses.

\section{Factors Associated With SP in Individual Nonoccluded Vessels}

In the univariate analysis for factors related to significant SP in MCAs, the highest-quartile WSSV was significantly associated with an SP $\geq 20 \%$ and an SPR $\geq 10 \% /$ year, whereas the highest-quartile SD was associated with an SPR $\geq 10 \% / y e a r$. No conventional cerebrovascular risk factor was associated with SP. After adjusting for factors associated with the initial SD, multivariate analysis showed that a WSSV in the highest quartile was the only prognostic factor for an SP $\geq 20 \%$ and an SPR $\geq 10 \%$ /year. In the univariate analysis of ICAs, a WSSV in the highest quartile was associated significantly with an SP $\geq 20 \%$ and an SPR $\geq 10 \% / y e a r$, whereas factors regarding the initial SD or cardiovascular risk factors were insignificant. In the multivariate analysis, a WSSV in the highest quartile was confirmed to be the predictive factor for an $\mathrm{SP} \geq 20 \%$ and an SPR $\geq 10 \% / y e a r$. In the univariate analysis of all individual vessels (MCAs and ICAs combined), a WSSV

TABLE 3. WSS and SP profiles for vessels*

\begin{tabular}{|c|c|c|c|}
\hline Variable & $\operatorname{ICA}(n=57)$ & $\operatorname{MCA}(n=38)$ & $p$ Value \\
\hline Diameter (mm) & $2.3 \pm 0.6$ & $1.7 \pm 0.6$ & $<0.001 \dagger$ \\
\hline MFV (cm/sec) & $70.4 \pm 45.3$ & $123.3 \pm 65.8$ & $<0.001 \dagger$ \\
\hline $\mathrm{Pl}$ & $0.7 \pm 0.2$ & $0.6 \pm 0.1$ & $0.002 \dagger$ \\
\hline $\operatorname{SR}\left(\sec ^{-1}\right)$ & $285.7 \pm 299.8$ & $377.7 \pm 307.7$ & 0.153 \\
\hline WSSV (dyne/cm²) & $12.3 \pm 8.5$ & $15.4 \pm 9.7$ & 0.109 \\
\hline Reynolds number & $\begin{array}{c}180.9 \pm 132.2 \\
(41.2-683.0)\end{array}$ & $\begin{array}{l}223.3 \pm 127.0 \\
(35.0-493.2)\end{array}$ & 0.084 \\
\hline Initial SD (\%) & $20.2 \pm 18.7$ & $42.7 \pm 21.9$ & $0.002 \dagger$ \\
\hline Follow-up SD (\%) & $20.2 \pm 25.1$ & $57.3 \pm 35.0$ & \\
\hline $\mathrm{SP}(\%)$ & $0.0 \pm 19.1$ & $14.7 \pm 23.1$ & $0.002 \dagger$ \\
\hline$S P \geq 20 \%$ & $7(12.3)$ & $15(39.5)$ & $0.002 \dagger$ \\
\hline SPR (\%/yr) & $0.2 \pm 15.3$ & $8.5 \pm 19.0$ & $0.027 \dagger$ \\
\hline $\mathrm{SPR} \geq 10 \% / \mathrm{yr}$ & $10(17.5)$ & $16(42.1)$ & $0.008 \dagger$ \\
\hline \multicolumn{4}{|c|}{$\begin{array}{l}\mathrm{PI}=\text { pulsatility index. } \\
\text { * } \text { Data are reported as mean } \pm \text { standard deviation (range) or number }(\%) \text { of } \\
\text { vessels }(n=95) \text {. } \\
\dagger \text { Statistically significant }(p<0.05) \text {. }\end{array}$} \\
\hline
\end{tabular}

in the highest quartile was significantly associated with an $\mathrm{SP} \geq 20 \%$ and an SPR $\geq 10 \% /$ year, whereas an SD in the highest quartile was associated with an $\mathrm{SP} \geq 20 \%$. Moreover, MCAs, more so than ICAs, were associated with an SP $\geq 20 \%$ and an SPR $\geq 10 \% / y e a r$. Multivariate analysis showed that a WSSV in the highest quartile was the independent prognostic factor for an $\mathrm{SP} \geq 20 \%$ and an SPR $\geq$ $10 \% / y e a r$. MCAs, more so than ICAs, remained significantly associated with an SP $\geq 20 \%$ and an SPR $\geq 10 \%$ / year (Table 4).

\section{Factors Associated With SP in Individual Hemispheres}

In the univariate analysis for factors related to significant SP in each hemisphere, an MWSSV in the highest quartile was significantly associated with an MSP $\geq$ $20 \%$ and an MSPR $\geq 10 \%$ /year, whereas hyperlipidemia was also associated with an MSP $\geq 20 \%$. No other conventional cerebrovascular risk factors or initial SD factors were significantly associated with SP. Multivariate analysis revealed that an MWSSV in the highest quartile was the only prognostic factor for an MSP $\geq 20 \%$ and an MSPR $\geq 10 \%$ year (Table 5). The durations of follow-up were comparable between the group with an MSP $\geq 20 \%$ and the group with an MSP $<20 \%$, and the group with an MSPR $<10 \% / y e a r$ had a longer follow-up duration than the group with an MSPR $\geq 10 \% / y e a r$. Furthermore, groups with significant hemispheric SP (MSP $\geq 20 \%$ and MSPR $\geq 10 \%$ /year) showed more frequent development of vascular complications than the groups without it. The times until complication development were comparable between the groups (Table 6).

\section{Factors Associated With Vascular Complications in Individual Hemispheres}

In the univariate analysis for factors related to the development of vascular complications in relevant hemispheres, an MWSSV in the highest quartile was significantly associated with the development of vascular complications. No other conventional cerebrovascular risk factors or initial SD factors had a significant association. Multivariate analysis revealed that an MWSSV in the highest quartile was the only predictor for vascular complications (Table 7).

\section{ROC Curve Analysis}

In ROC curve analysis for MCAs, a WSSV in the highest quartile predicted an SP $\geq 20 \%$ with a sensitivity of $73.3 \%$ and a specificity of $69.6 \%$, and an SPR $\geq 10 \% /$ year with a sensitivity of $81.3 \%$ and a specificity of $68.2 \%$. For ICAs, a WSSV in the highest quartile predicted an SP $\geq$ $20 \%$ with a sensitivity of $71.4 \%$ and a specificity of $64.0 \%$, and an SPR $\geq 10 \% / y e a r$ with a sensitivity of $80.0 \%$ and a specificity of $64.0 \%$. For all individual vessels combined, a WSSV in the highest quartile predicted an SP $\geq 20 \%$ with a sensitivity of $81.8 \%$ and a specificity of $71.2 \%$, and an SPR $\geq 10 \% /$ year with a sensitivity of $80.8 \%$ and a specificity of $71.0 \%$. For each hemisphere, an MWSSV in the highest quartile predicted an MSP $\geq 20 \%$ with a sensitivity of $76.5 \%$ and a specificity of $69.2 \%$, and an MSPR $\geq$ $10 \% / y e a r$ with a sensitivity of $63.6 \%$ and a specificity of $58.7 \%$ (Fig. 1). 


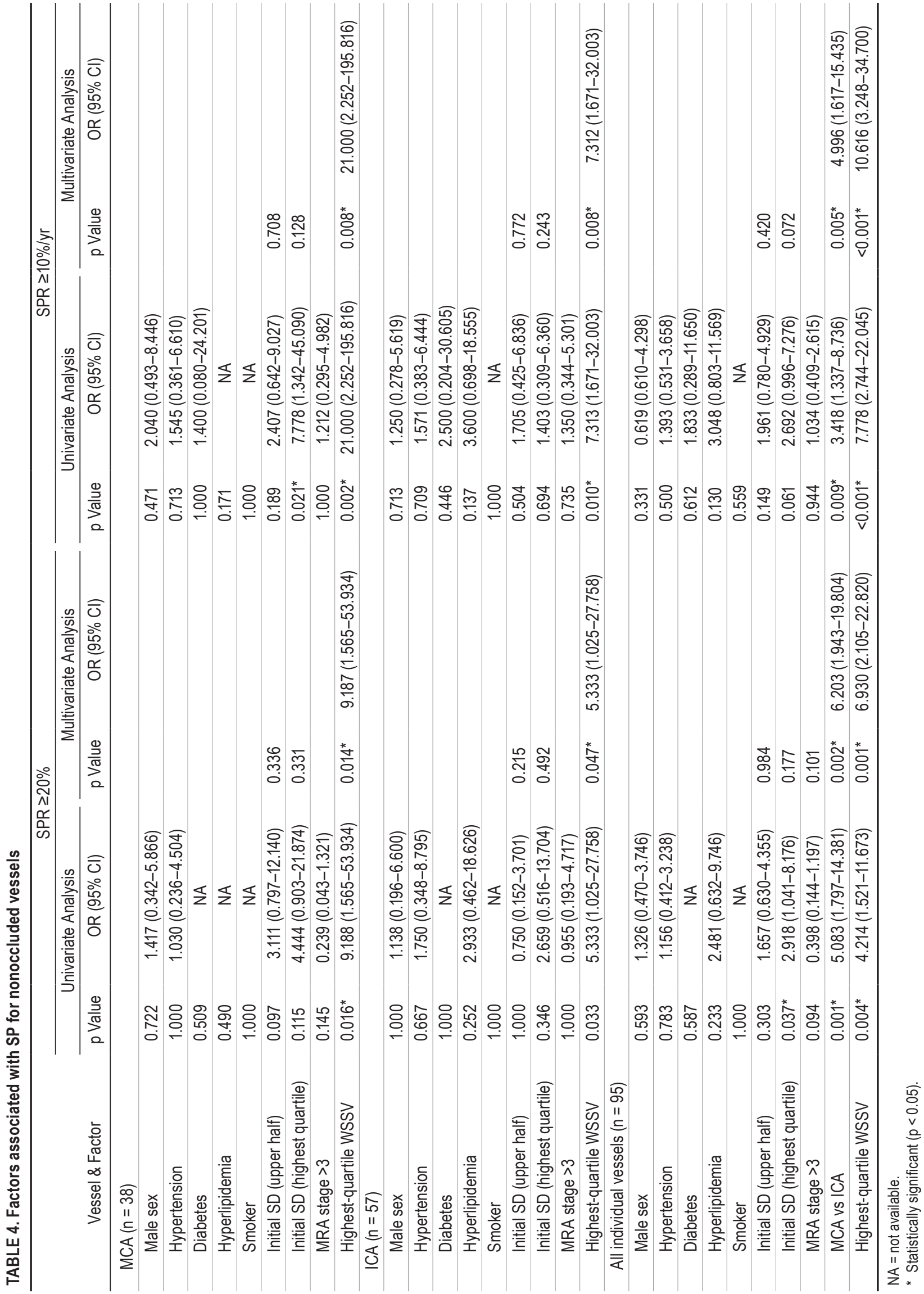




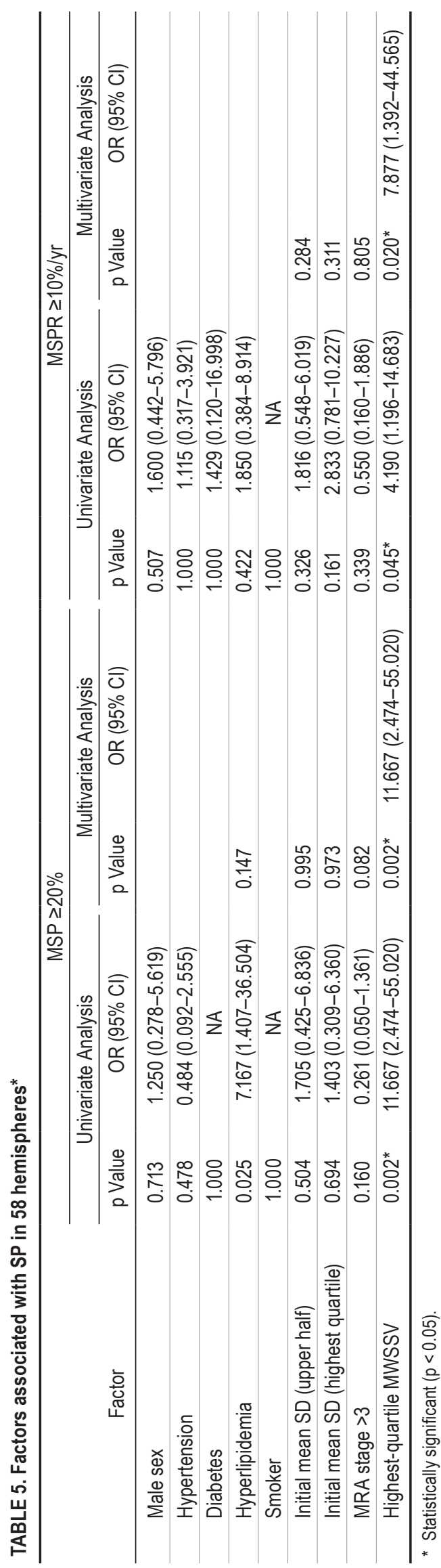

\section{Discussion}

In patients with MMD, before vessels became occluded, a WSSV in the highest quartile was associated with the absolute magnitude (SP) and rate (SPR) of SP in each vessel and also with the absolute magnitude (MSP) and rate (MSPR) of SP in each hemisphere. Furthermore, significant SP was highly associated with the development of symptoms, and a hemispheric MWSSV in the highest quartile was an independent predictor of vascular complications in the relevant hemispheres. This relationship was independent of initial SD, MR stage of MMD, duration of follow-up, and other vascular risk factors. This result might be explained by an altered vascular remodeling response to elevated WSS; thus, the WSSV according to TCD might be an important indicator of MMD progression.

When the vessel is not stenosed and blood flow is undisturbed, WSS normally keeps endothelial cells in a stable state by downregulating inflammatory cytokines (e.g., interleukin 1, interleukin 4, and tumor necrosis factor- $\alpha$ ) and growth factors (e.g., platelet-derived growth factor, fibroblast growth factor-2, and insulin-like growth factorI). $4,8,16,23$ This noninflammatory/nonproliferative state results in the suppression of basement membrane-degrading MMPs (MMP-2 and MMP-9) and inhibition of smooth-

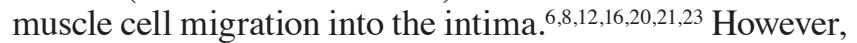
in a stenosed vessel, a decrease in the diameter likely increases the WSS. Elevated WSS causes endothelial cells and smooth-muscle cells to significantly overexpress MMP-2 and MMP-9, consequently inducing a disproportional increase of MMPs over TIMPs and thereby degrading the extracellular matrix and promoting the migration and proliferation of vascular smooth-muscle cells, which results in adaptive vascular remodeling. ${ }^{26}$

In patients with MMD, SP is accompanied by fibrocellular thickening of the intima with an increased number of smooth-muscle cells, undulation of the elastic lamina, and attenuation of the media, ${ }^{16,25}$ which implies a possibility that the altered behaviors of MMPs and TIMPs play a significant pathophysiological role in the SP of MMD. There have been numerous studies regarding the altered expression of MMPs and TIMPs in patients with MMD. Overexpression of MMP-92,5,13 and reduced expression of MMP-3, TIMP-1, and TIMP-2, $2,13,30$ have been documented in patients with MMD. Genetic studies of familial MMD have found polymorphisms in the MMP-3 gene ${ }^{19}$ and the TIMP-2 promoter gene. ${ }^{14}$ These alterations in MMP expression/function might result in immoderate extracellular matrix degradation/deposition and smooth-muscle cell proliferation/migration in response to elevated WSS. The excessive thickening of the intima and progressive vascular narrowing in patients with MMD are possible results of this dysregulation in the vascular remodeling response to high WSS in the terminal ICA and its proximal branches, where the flow is considerably fast and branches are in a very acute angle (proximal MCA). Considering that the initial SD was not significantly associated with SP in our study, WSS elevation might be an independent driving force behind the progression of MMD and not simply a consequence of vessel stenosis.

Our finding that MCAs were more associated with SP than ICAs is also interesting. Considering the relatively 
TABLE 6. Comparison of follow-up duration, vascular complication frequency, and time to complication development in groups with and those without significant SP*

\begin{tabular}{|c|c|c|c|c|c|c|}
\hline Factor & $\begin{array}{l}\text { Hemispheres w/ } \\
\text { MSP } \geq 20 \%\end{array}$ & $\begin{array}{l}\text { Hemispheres } \\
\text { w/ MSP <20\% }\end{array}$ & $p$ Value & $\begin{array}{l}\text { Hemispheres w/ } \\
\text { MSPR } \geq 10 \% / y r\end{array}$ & $\begin{array}{l}\text { Hemispheres w/ } \\
\text { MSPR < } 10 \% / y r\end{array}$ & $\mathrm{p}$ Value \\
\hline Follow-up duration (mos) & $22.4 \pm 10.6$ & $27.3 \pm 14.0$ & 0.231 & $19.8 \pm 9.3$ & $28.2 \pm 14.0$ & $0.021 \dagger$ \\
\hline Vascular complication (\%) & 80.0 & 4.2 & $<0.001 \dagger$ & 50.0 & 8.7 & $<0.001 \dagger$ \\
\hline Time to complication development (mos) & $22.1 \pm 10.7$ & $21.0 \pm 7.1$ & 0.893 & $18.8 \pm 10.3$ & $26.5 \pm 7.7$ & 0.217 \\
\hline
\end{tabular}

* Data are reported as mean \pm standard deviation or percentage.

$\dagger$ Statistically significant $(p<0.05)$.

TABLE 7. Factors associated with vascular complication for 58 hemispheres

\begin{tabular}{|c|c|c|c|c|}
\hline \multirow[b]{3}{*}{ Factor } & \multicolumn{4}{|c|}{ Vascular Complication } \\
\hline & \multicolumn{2}{|c|}{ Univariate Analysis } & \multicolumn{2}{|c|}{ Multivariate Analysis } \\
\hline & $\mathrm{p}$ Value & $\mathrm{OR}(95 \% \mathrm{Cl})$ & $\mathrm{p}$ Value & OR $(95 \% \mathrm{Cl})$ \\
\hline Male sex & 0.439 & $2.000(0.482-8.306)$ & & \\
\hline Hypertension & 1.000 & $0.857(0.195-3.764)$ & & \\
\hline Diabetes & 1.000 & NA & & \\
\hline Hyperlipidemia & 0.702 & $0.651(0.071-5.968)$ & & \\
\hline Smoker & 1.000 & NA & & \\
\hline Initial mean SD (upper half) & 0.164 & $2.758(0.636-11.956)$ & 0.082 & \\
\hline Initial mean SD (highest quartile) & 0.233 & $2.533(0.598-10.738)$ & 0.287 & \\
\hline MRA stage $>3$ & 0.160 & $0.250(0.048-1.301)$ & 0.069 & \\
\hline Highest-quartile MWSSV & $0.002^{*}$ & $11.667(2.474-55.020)$ & $0.002^{*}$ & $15.881(2.719-82.770)$ \\
\hline
\end{tabular}

* Statistically significant $(p<0.05)$.
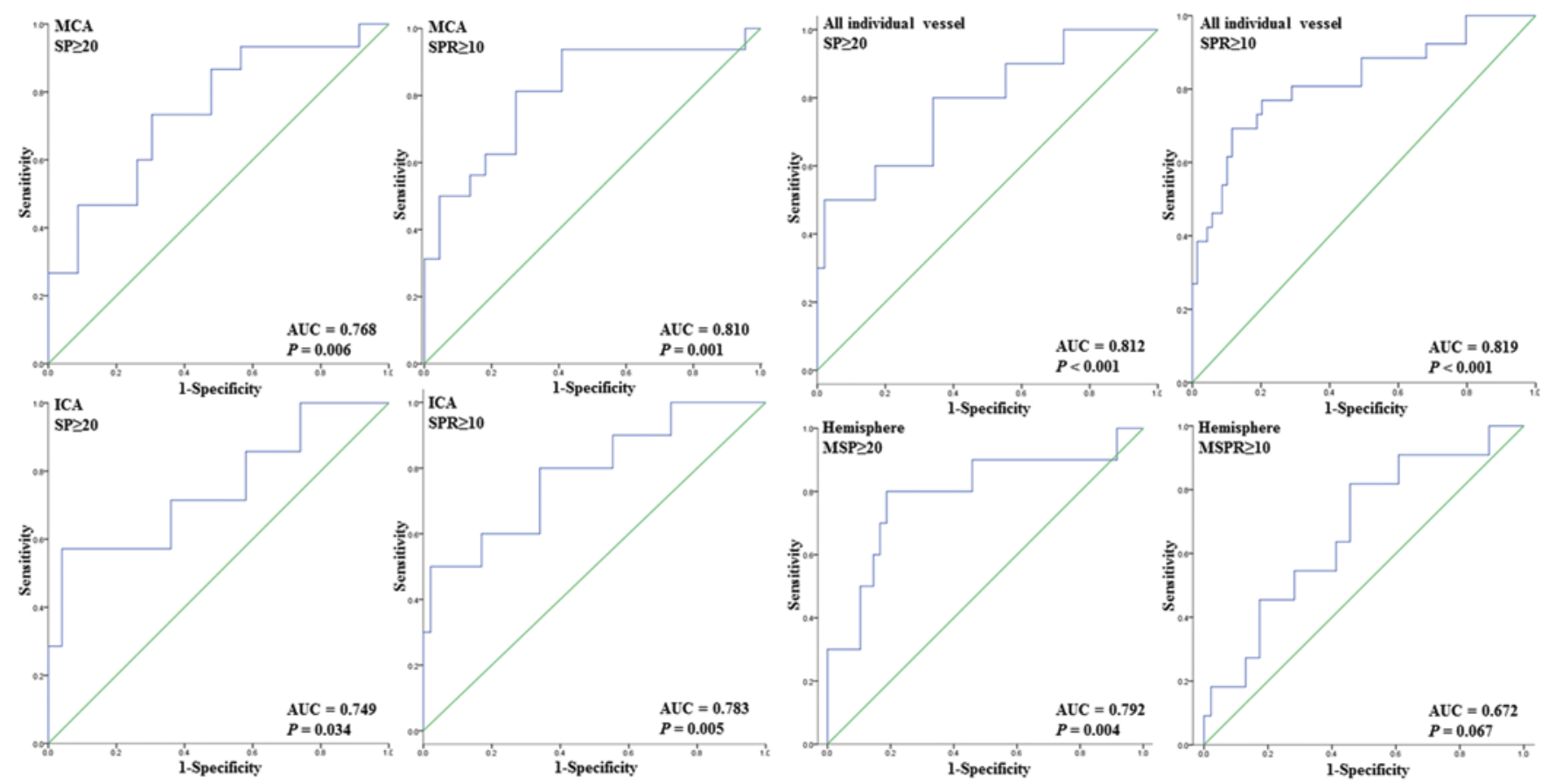

FIG. 1. Predictive power of a high WSS for SP:ROC curves. ROC curves of WSSVs for parameters of significant SP (SP $\geq 20$ and $\mathrm{SPR} \geq 10$ for ICAs/MCAs/all vessels, and MSP $\geq 20$ and MSPR $\geq 10$ for hemispheric means) are shown. Figure is available in color online only. 
early disease stages of the study population, we can infer that the proximal MCA might usually be involved before the distal ICA in the early stage of MMD. Additional studies aimed at replicating our findings and determining the underlying mechanism should be conducted.

There are several limitations of our study. First, we indirectly estimated that the SR of blood flow would be Newtonian and did not take vessel curvature into account. Therefore, we adopted an indirect term, WSSV, to indicate the relative magnitude of the actual WSS. Computational flow dynamic studies using 3D angiography are necessary to evaluate the actual WSS at the site of interest. This direct estimation is especially important in investigating WSS profiles of turbulent flow characteristics, such as those of intracranial aneurysms. ${ }^{27}$ However, in clinical contexts in which laminar flow could be assumed, numerous studies sonographically measured the relative magnitude of WSS and performed quantitative analysis to establish its pathophysiological role in the development of various vascular diseases. ${ }^{10,11}$ The parabolic velocity profile was assumed in those studies, and velocity at the centerline of flow was evaluated. Considering that the ophthalmic segment of the ICA and the proximal $\mathrm{M}_{1}$ of the MCA are relatively large and straight, that the flow characteristics in this study were laminar with uniformly low Reynolds numbers, and that the MFV can be estimated precisely with $\mathrm{TCD},{ }^{1}$ the relative magnitude of WSS evaluated in this study might have sufficient validity. Second, we used TOF sequences to estimate SDs, which might have overestimated the SDs in cases of highly stenosed vessels. However, the highest initial SD was $72.4 \%$ (the highest follow-up SD was $83.4 \%$ ), and only 2 vessels had an SD > 70\% (6 vessels had a follow-up SD $\geq 70 \%$ ), which indicates that the initial and follow-up SDs might have been evaluated appropriately in most cases. Third, the number of patients in this study was small, which might weaken the statistical certainty. This small study population might be related to the rarity of MMD and the high frequency of surgical intervention. Fourth, TCD is an evaluation tool that depends highly on the technician. In addition, our retrospective design and relatively short follow-up duration (median 23 months) might also be limitations of our study. Prospective studies aimed at confirming and extending the retrospective findings of different Doppler laboratories might be warranted.

\section{Conclusions}

In patients with MMD, elevated WSS is independently associated with SP and the development of vascular complications. Evidence from large prospective studies or from direct measurement of WSS is needed before applying these values in clinical practice.

\section{References}

1. Aaslid R, Markwalder TM, Nornes H: Noninvasive transcranial Doppler ultrasound recording of flow velocity in basal cerebral arteries. J Neurosurg 57:769-774, 1982

2. Achrol AS, Guzman R, Lee M, Steinberg GK: Pathophysiology and genetic factors in moyamoya disease. Neurosurg Focus 26(4):E4, 2009

3. Box FM, van der Geest RJ, Rutten MC, Reiber JH: The influence of flow, vessel diameter, and non-Newtonian blood vis- cosity on the wall shear stress in a carotid bifurcation model for unsteady flow. Invest Radiol 40:277-294, 2005

4. Chen BP, Li YS, Zhao Y, Chen KD, Li S, Lao J, et al: DNA microarray analysis of gene expression in endothelial cells in response to 24-h shear stress. Physiol Genomics 7:55-63, 2001

5. Fujimura M, Watanabe M, Narisawa A, Shimizu H, Tominaga T: Increased expression of serum matrix metalloproteinase-9 in patients with moyamoya disease. Surg Neurol 72:476-480, 2009

6. Garanich JS, Pahakis M, Tarbell JM: Shear stress inhibits smooth muscle cell migration via nitric oxide-mediated downregulation of matrix metalloproteinase-2 activity. Am J Physiol Heart Circ Physiol 288:H2244-H2252, 2005

7. Gnasso A, Carallo C, Irace C, Spagnuolo V, De Novara G, Mattioli PL, et al: Association between intima-media thickness and wall shear stress in common carotid arteries in healthy male subjects. Circulation 94:3257-3262, 1996

8. Houkin K, Ito M, Sugiyama T, Shichinohe H, Nakayama N, Kazumata K, et al: Review of past research and current concepts on the etiology of moyamoya disease. Neurol Med Chir (Tokyo) 52:267-277, 2012

9. Houkin K, Nakayama N, Kuroda S, Nonaka T, Shonai T, Yoshimoto T: Novel magnetic resonance angiography stage grading for moyamoya disease. Cerebrovasc Dis 20:347354,2005

10. Irace C, Carallo C, Crescenzo A, Motti C, De Franceschi MS, Mattioli PL, et al: NIDDM is associated with lower wall shear stress of the common carotid artery. Diabetes 48:193197, 1999

11. Jeong SK, Rosenson RS: Shear rate specific blood viscosity and shear stress of carotid artery duplex ultrasonography in patients with lacunar infarction. BMC Neurol 13:36, 2013

12. Johnson C, Galis ZS: Matrix metalloproteinase-2 and -9 differentially regulate smooth muscle cell migration and cellmediated collagen organization. Arterioscler Thromb Vasc Biol 24:54-60, 2004

13. Kang HS, Kim JH, Phi JH, Kim YY, Kim JE, Wang KC, et al: Plasma matrix metalloproteinases, cytokines and angiogenic factors in moyamoya disease. J Neurol Neurosurg Psychiatry 81:673-678, 2010

14. Kang HS, Kim SK, Cho BK, Kim YY, Hwang YS, Wang KC: Single nucleotide polymorphisms of tissue inhibitor of metalloproteinase genes in familial moyamoya disease. Neurosurgery 58:1074-1080, 2006

15. Kim JE, Kim KM, Kim JG, Kang HS, Bang JS, Son YJ, et al: Clinical features of adult moyamoya disease with special reference to the diagnosis. Neurol Med Chir (Tokyo) 52:311-317, 2012

16. Kronenburg A, Braun KP, van der Zwan A, Klijn CJ: Recent advances in moyamoya disease: pathophysiology and treatment. Curr Neurol Neurosci Rep 14:423, 2014

17. Kuroda S, Hashimoto N, Yoshimoto T, Iwasaki Y: Radiological findings, clinical course, and outcome in asymptomatic moyamoya disease: results of multicenter survey in Japan. Stroke 38:1430-1435, 2007

18. Lee YS, Jung KH, Roh JK: Diagnosis of moyamoya disease with transcranial Doppler sonography: correlation study with magnetic resonance angiography. J Neuroimaging 14:319323, 2004

19. Li H, Zhang ZS, Liu W, Yang WZ, Dong ZN, Ma MJ, et al: Association of a functional polymorphism in the MMP-3 gene with moyamoya disease in the Chinese Han population. Cerebrovasc Dis 30:618-625, 2010

20. Mason DP, Kenagy RD, Hasenstab D, Bowen-Pope DF, Seifert RA, Coats S, et al: Matrix metalloproteinase-9 overexpression enhances vascular smooth muscle cell migration and alters remodeling in the injured rat carotid artery. Circ Res 85:1179-1185, 1999 
21. Newby AC: Matrix metalloproteinases regulate migration, proliferation, and death of vascular smooth muscle cells by degrading matrix and non-matrix substrates. Cardiovasc Res 69:614-624, 2006

22. Nicoletto HA, Burkman MH: Transcranial Doppler series part II: performing a transcranial Doppler. Am J Electroneurodiagnostic Technol 49:1-27, 2009

23. Palumbo R, Gaetano C, Melillo G, Toschi E, Remuzzi A, Capogrossi MC: Shear stress downregulation of platelet-derived growth factor receptor-beta and matrix metalloprotease- 2 is associated with inhibition of smooth muscle cell invasion and migration. Circulation 102:225-230, 2000

24. Ryoo S, Cha J, Kim SJ, Choi JW, Ki CS, Kim KH, et al: High-resolution magnetic resonance wall imaging findings of Moyamoya disease. Stroke 45:2457-2460, 2014

25. Scott RM, Smith ER: Moyamoya disease and moyamoya syndrome. N Engl J Med 360:1226-1237, 2009

26. Sho E, Sho M, Singh TM, Nanjo H, Komatsu M, Xu C, et al: Arterial enlargement in response to high flow requires early expression of matrix metalloproteinases to degrade extracellular matrix. Exp Mol Pathol 73:142-153, 2002

27. Shojima M, Oshima M, Takagi K, Torii R, Hayakawa M, Katada K, et al: Magnitude and role of wall shear stress on cerebral aneurysm: computational fluid dynamic study of 20 middle cerebral artery aneurysms. Stroke 35:2500-2505, 2004

28. Sloan MA, Alexandrov AV, Tegeler CH, Spencer MP, Caplan LR, Feldmann E, et al: Assessment: transcranial Doppler ultrasonography: report of the Therapeutics and Technology Assessment Subcommittee of the American Academy of Neurology. Neurology 62:1468-1481, 2004

29. Takeuchi S, Karino T: Flow patterns and distributions of fluid velocity and wall shear stress in the human internal carotid and middle cerebral arteries. World Neurosurg 73:174-185, e27, 2010

30. Wang X, Zhang Z, Liu W, Xiong Y, Sun W, Huang X, et al: Impacts and interactions of PDGFRB, MMP-3, TIMP-2, and RNF213 polymorphisms on the risk of moyamoya disease in Han Chinese human subjects. Gene 526:437-442, 2013

31. Zanette EM, Fieschi C, Bozzao L, Roberti C, Toni D, Argentino $\mathrm{C}$, et al: Comparison of cerebral angiography and transcranial Doppler sonography in acute stroke. Stroke 20:899-903, 1989

\section{Disclosures}

The authors report no conflict of interest concerning the materials or methods used in this study or the findings specified in this paper.

\section{Author Contributions}

Conception and design: Jung, KJ Lee, ST Lee, Roh. Acquisition of data: WJ Lee. Analysis and interpretation of data: WJ Lee, Kim, Chu. Drafting the article: WJ Lee. Critically revising the article: SK Lee, Roh. Reviewed submitted version of manuscript: all authors. Approved the final version of the manuscript on behalf of all authors: Jung. Statistical analysis: WJ Lee. Administrative/technical/material support: Kim, ST Lee. Study supervision: Jung, Chu, SK Lee.

\section{Correspondence}

Keun-Hwa Jung, Department of Neurology, Seoul National University Hospital, 101 Daehangno, Jongnogu, Seoul 110-744, South Korea.email: jungkh@gmail.com. 